\title{
A xenogeneic-free system generating functional human gut organoids from pluripotent stem cells
}

\author{
Hajime Uchida,,${ }^{1,2}$ Masakazu Machida, ${ }^{3}$ Takumi Miura, ${ }^{3}$ Tomoyuki Kawasaki, ${ }^{3}$ Takuya Okazaki, ${ }^{4}$ \\ Kengo Sasaki, ${ }^{1,2}$ Seisuke Sakamoto, ${ }^{1}$ Noriaki Ohuchi, ${ }^{2}$ Mureo Kasahara, ${ }^{1}$ Akihiro Umezawa, ${ }^{3}$ \\ and Hidenori Akutsu ${ }^{3,5}$ \\ 'Transplantation Centre, National Centre for Child Health and Development, Tokyo, Japan. ${ }^{2}$ Department of Surgery, \\ Tohoku University, Sendai, Japan. ${ }^{3}$ Department of Reproductive Biology, Centre for Regenerative Medicine, National \\ Research Institute for Child Health and Development, Tokyo, Japan. ${ }^{4}$ Life Science Department, Research and \\ Development Center, Dai Nippon Printing Co., Ltd., Tokyo, Japan. ${ }^{5}$ Department of Stem Cell Research, Fukushima \\ Medical University, Fukushima, Japan
}

Functional intestines are composed of cell types from all 3 primary germ layers and are generated through a highly orchestrated and serial developmental process. Directed differentiation of human pluripotent stem cells (hPSCs) has been shown to yield gut-specific cell types; however, these structures do not reproduce critical functional interactions between cell types of different germ layers. Here, we developed a simple protocol for the generation of mature functional intestinal organoids from hPSCs under xenogeneic-free conditions. The stem cell-derived gut organoids produced here were found to contain distinct types of intestinal cells, including enterocytes, goblet cells, Paneth cells, and enteroendocrine cells, that were derived from all 3 germ layers; moreover, they demonstrated intestinal functions, including peptide absorption, and showed innervated bowel movements in response to stimulation with histamine and anticholinergic drugs. Importantly, the gut organoids obtained using this xenogeneic-free system could be stably maintained in culture for prolonged periods and were successfully engrafted in vivo. Our xenogeneic-free approach for generating gut organoids from hPSCs provides a platform for studying human intestinal diseases and for pharmacological testing

Conflict of interest: The authors have declared that no conflict of interest exists.

Submitted: January 12, 2016 Accepted: November 22, 2016 Published: January 12, 2017

Reference information: JCI Insight. 2017;2(1):e86492. doi:10.1172/jci.insight.86492.

\section{Introduction}

The intestine is a complex organ derived from all 3 germ layers. It is composed of intestinal epithelium containing absorptive secretory cells and resident adult stem cells, intestinal lamina propria, submucosa, muscularis mucosa, and serosa; each of these cell layers is characterized by specific cellular compositions and distinct functions $(1,2)$. The development of the gastrointestinal tube involves a series of complex mutual interactions among the mesenchyme, endodermal epithelium, and developing ectodermal enteric nervous system $(3,4)$.

Human pluripotent stem cells (hPSCs) can be induced to differentiate into various cell types by manipulating developmental signals, first to generate germ layers and then to produce distinct cell types, such as pancreatic $\beta$ cells and dopaminergic neurons (5-8). Recently, it was reported that PSCs or isolated tissue progenitors could be induced to generate intestine-like 3-dimensional "organoids" (9-11). However, these gut epithelial structures were obtained using sequential induction with a panel of growth factors to first generate endoderm, while suppressing formation of other germ layers, and then to induce differentiation into specific intestinal cell types (9). Therefore, these protocols do not mimic the functional interactions of the 3 germ layers that occur during organogenesis in vivo. Moreover, in these studies, differentiation occurred under xenogeneic conditions, which complicates the possible use of these structures in clinical applications. Here, we demonstrate efficient production of functional gut organoids from hPSCs using xenogeneic-free (XF) conditions. These organoids contained differentiated epithelial cells, including nutrient-absorbing enterocytes, and secretory lineage cells, goblet cells, Paneth cells, and enteroendocrine cells, together with innervated smooth muscle. Thus, they represent a downsized human intestinal organ (gut organoid) suitable for pharmacological testing. 
A Generating gut organoids on a single platform

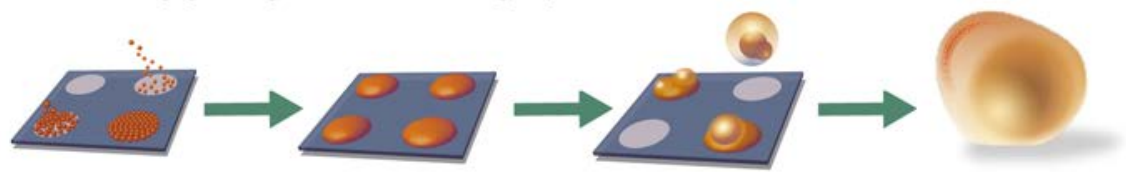

\begin{tabular}{|c|c|c|c|}
\hline XF-KSR(-) & \multicolumn{3}{|c|}{ IGF- $1 /$ heregulin- $1 \beta / \mathrm{bFGF}$ in XF-KSR based medium } \\
\hline D0-D3 & D4- & D30- & D60 \\
\hline $\begin{array}{l}\text { assembling \& } \\
\text { patterning }\end{array}$ & $\begin{array}{l}\text { formation of hemispheric dome-like } \\
\text { structures due to piling-up of cells }\end{array}$ & emerging out & gut_like peristalsis \\
\hline
\end{tabular}

B
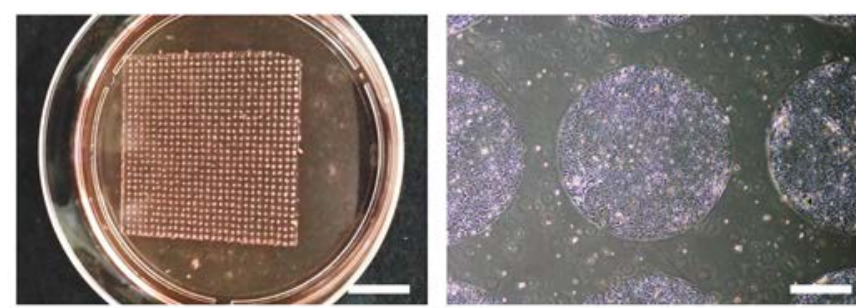

C

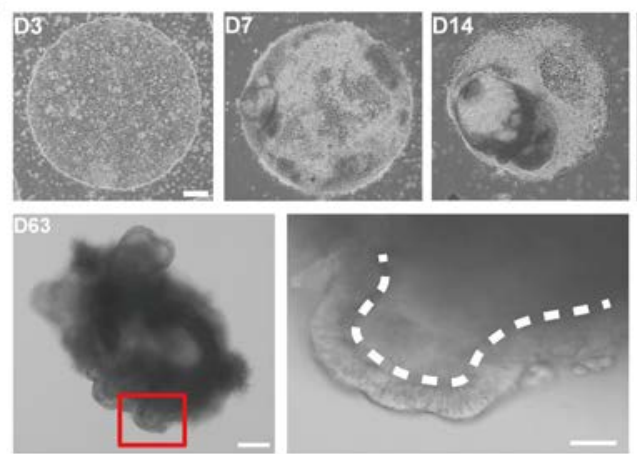

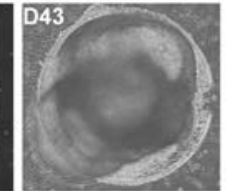

D

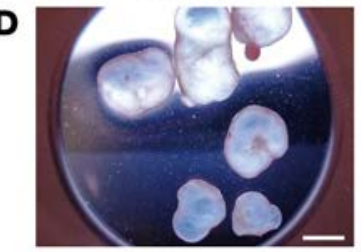

Figure 1. Generation of peristaltic gut organoids from human pluripotent stem cells on a patterning substrate. (A) Illustration of gut organoid generation from human pluripotent stem cells cultured on a single device. (B) A micropatterned substrate for cell growth was produced by coating a small mesh net, 1,500 $\mu \mathrm{m}$ in diameter (left panel), with a bioactive polymer (scale bar: $15 \mathrm{~mm}$ ). The right panel shows that human embryonic stem cells attached and grew only within each zone (scale bar: $500 \mu \mathrm{m}$ ). (C) Time course of organoid growth in culture. The organoid at day 63 is surrounded by intestinal columnar epithelium layers adjacent to mesenchymal tissues; these epithelial layers are delineated by the dotted white line (high-magnification view of the area within the red box). Scale bar: $200 \mu \mathrm{m}$ (top row and right bottom); $50 \mu \mathrm{m}$ (left bottom). (D) Six organoids that exhibited gut-like motor activity were maintained in culture at day 129 and did not coalesce. Scale bar: $5 \mathrm{~mm}$.

\section{Results}

Gut organoids self-organize on a patterning substrate. Tissue self-organization has three major steps: self-assembly, self-patterning, and self-morphogenesis (12). We applied this three-step concept to inducing gut morphogenesis via a cellular patterning-bulging phase and a self-morphogenesis phase (Figure 1A). In the first step, human embryonic stem cells (hESCs) or induced hPSCs (hiPSCs) that had been cultured in XF medium (13) were dissociated and placed on a circle-patterned glass surface (14) in differentiation medium. This surface was coated with the polyethylene glycol micropatterning substrate CytoGraph (Figure 1B), which stimulates cell assemblage. The cells accumulated in restricted areas and formed hemispheric dome-like structures after 7 days (Figure 1C). Subsequently, enlarged cavitated structures formed; these exhibited epithelial folding and cyst-like protrusions of a self-organized mass and by day 20 were lined with cuboidal epithelial cells. By day 30 , self-organized cystic spheroids began to detach from the plate. These spheroids mostly belonged to two morphological types: simple cystic forms with thin cellular walls and bicomponent spheroids with solid and partially cystic protrusions (Figure 1C and Supplemental Figure 1; supplemental material available online with this article; doi:10.1172/jci.insight.86492DS1). Remarkably, the bicomponent spheroids exhibited contractile movements; these spheroids comprised $4 \%$ ( 32 of $791, n=3$ ) of all detached organoids, and they were maintained over an extended period (Figure 1D and Supplemental Video 1). This suggests that the organoids consisted of cell types that originated from different germ layers and that were assembled into a functional cellular network.

Next, we examined expression of germ layer markers at different differentiation time points. In the early differentiation phase, growing patterned cells expressed markers of definitive endoderm, namely, FOXA2, SOX17, and CXCR4, and of early endoderm and mesoderm, namely, GATA4, GATA6, and T (Brachyury) (Figure 2A); their relative levels of expression increased in the hemispheric dome-like structures at day 14 (Figure 1A and Figure 2A). As differentiation progressed, the level of CXCR4 expression fell at day 21, a finding consistent with the reported downregulation of gene expression during hindgut formation in mouse embryo development (15). The level of the hindgut marker CDX2 increased during early growth and was maintained at a relatively high level as differentiation progressed (Figure 2A). The 
A

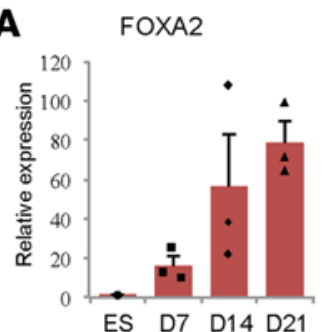

CDX2

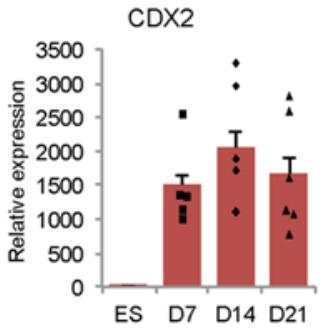

SoX17

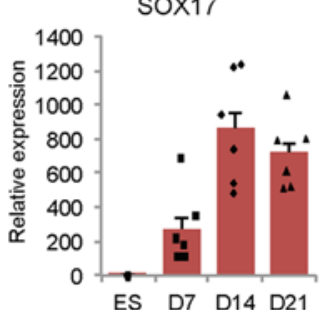

OCT4
CXCR4

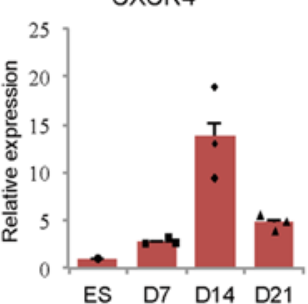

$\mathrm{T}$ (Brachyury)

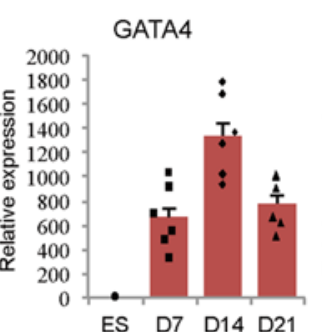

$\begin{array}{llll}\text { DS } & \text { D14 D21 }\end{array}$

SOX1
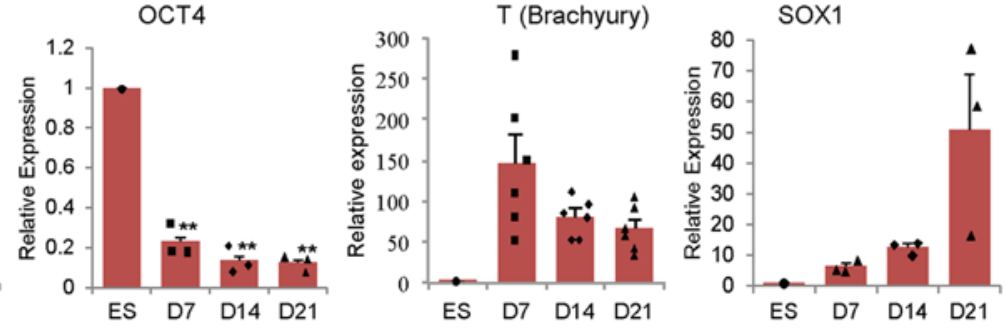

B
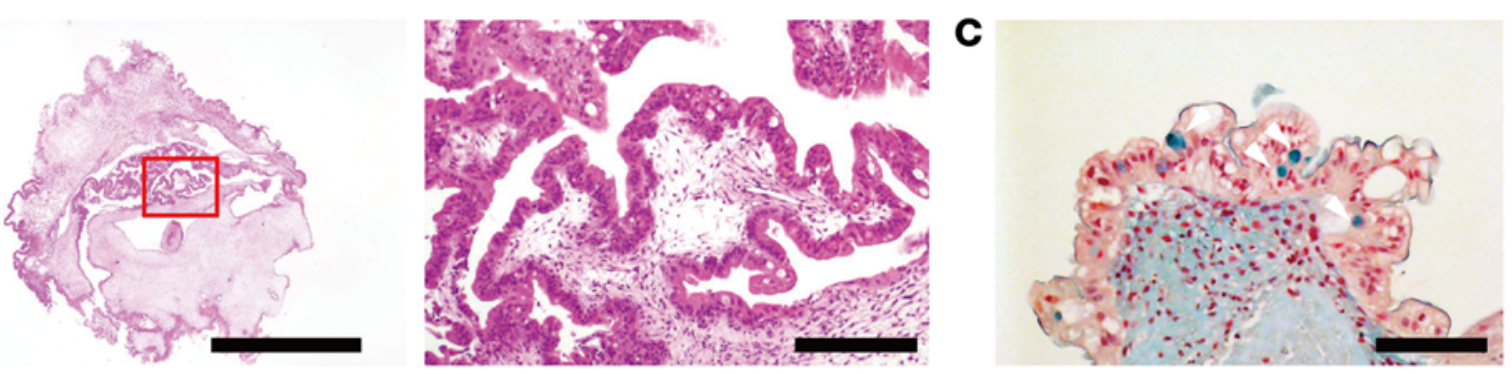

D

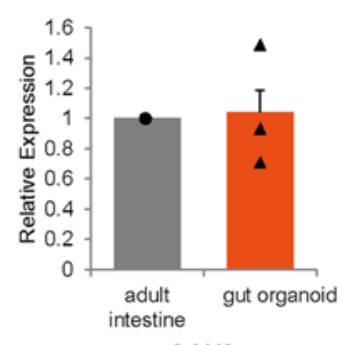

Sox9

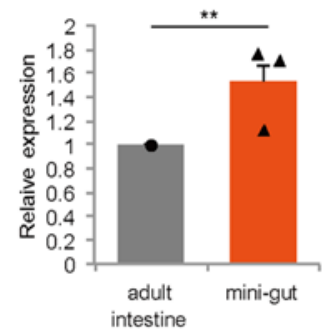

CD34

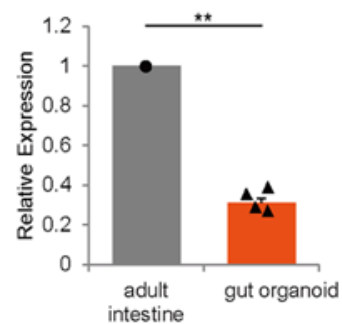

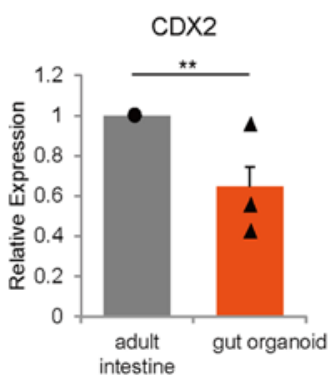

CGA

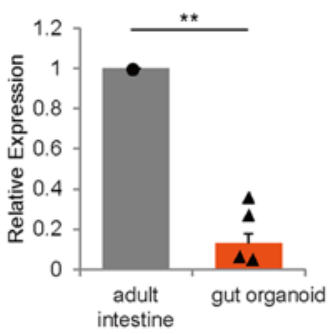

CKIT

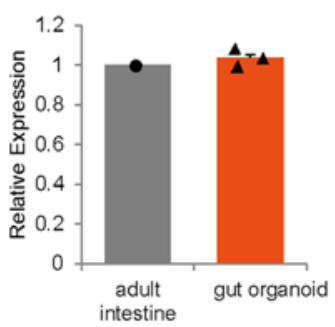

VILLIN

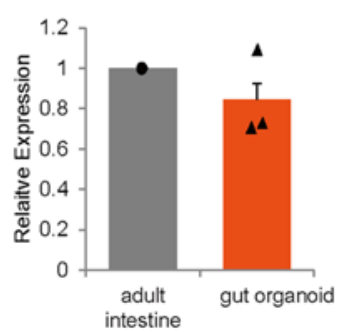

Lactase

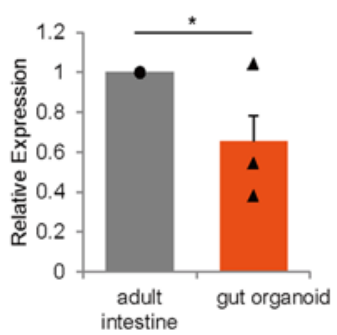

PGP9.5

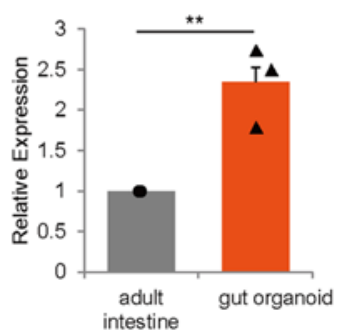

GATA6

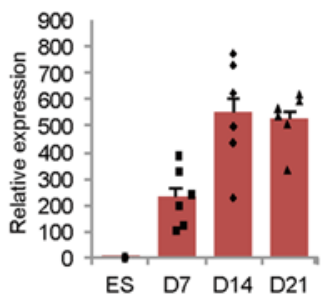

ES $\quad$ D7 $\quad$ D14 D21

ES $\quad$ 7
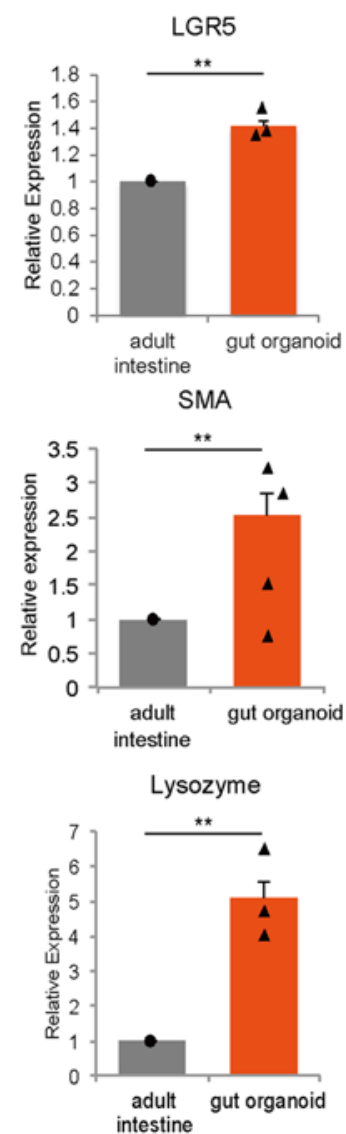

Figure 2. Characterization of developing gut organoids. (A) Analysis of expression of cell biomarker genes in differentiated human embryonic stem cells (hESCs) (SEES1 cells). Values were normalized against GAPDH. The data are reported as mean \pm SEM. Statistically significant differences were identified between embryonic stem (ES) vs. day 7, ES vs. day 14, and ES vs. day 21 using Student's $t$ test $\left({ }^{* *} P<0.01\right)(n=3-6)$. (B) H\&E staining of organoids with 
peristaltic movement at day 100. Scale bar: $2 \mathrm{~mm}$ (left); $200 \mu \mathrm{m}$ (right). (C) Alcian Blue staining of differentiated organoids (day 60) indicating goblet-like cells (white arrowheads). Scale bar: $100 \mu \mathrm{m}$. (D) Relative expression of cell biomarker genes in differentiated day 50 organoids and human adult small intestine. Statistical analysis was performed using a $t$ test or a Mann-Whitney rank-sum test $\left({ }^{*} P<0.05\right.$, $\left.{ }^{* *} P<0.01\right)$. The data are reported as means $(\%) \pm$ SEM and were obtained from 3 independent experiments $(n=3-4)$.

expression of the neural progenitor marker SOX1 increased at a relatively late stage (day 21) compared with other germ layer markers (Figure 2A). By contrast, the pluripotent cell marker OCT4 showed significant downregulation during early differentiation. Thus, our protocol promoted hESC differentiation into definitive endoderm and hindgut by day 21 , with the formation of hemispheric dome-like structures. During subsequent differentiation, cell accumulations formed cavitated structures and detached from the substratum (Figure 1C). Single gut-like epithelial layers exhibited partial multiple folds (Supplemental Video 1). H\&E staining of paraffin-embedded organoids that exhibited contractile movements showed that the gut architecture consisted of mucosa and submucosa (Figure 2B). The intestinal mucosa is formed by the epithelium and lamina propria; the intestinal epithelium consists of two cell types, absorptive enterocytes and secretory lineage cells, including mucus-producing goblet cells, enteroendocrine cells, and Paneth cells (1). Alcian Blue staining, which identifies acidic structures, showed the presence of purple-stained mucopolysaccharide-containing goblet cells in the epithelium of the organoids (Figure 2C). Interestingly, the relative expression of some late differentiation gene markers in mature organoids (day 50) was similar to that of an adult intestine (Figure 2D). As the intestinal epithelium matured, $C D X 2$, an intestinal transcription factor; $E C A D$, an epithelial cell-specific E-cadherin; and a brush border-specific villin were expressed in gut organoids at the same level as in the adult intestine. Immunostaining analysis showed that the villin protein localized to the apical surface of the epithelium and that CDX2 was present in the epithelial layer (Figure 3A). Transmission electron microscopy (TEM) of the organoids revealed the occurrence of a brush border in the apical microvilli (Figure 3B). Paneth cells were identified by staining for the Paneth cell-specific protein, defensin $\alpha-6$ (DEFA6) (Figure 3A), and were shown to contain secretory granules by TEM (Figure 3B). H\&E and Alcian Blue staining identified resident goblet cells in the epithelial layers, and their identity was confirmed by mucin-2 (MUC2) staining (Figure 3A) and by the presence of mucin granules under TEM (Figure 3B). The expression of chromogranin A (CGA), an epithelial enteroendocrine cell marker, was lower in gut organoids than in the adult healthy intestine (Figure 2D); however, immunostaining for ECAD and CGA showed the presence of both proteins in the epithelium (Figure 3A). The putative intestinal stem cell protein marker LGR5 was coexpressed with CDX2 in gut organoids (Figure 3A); it was also expressed during gut organogenesis in SEES3 cells (hESCs) transfected with EGFP under the LGR5 promoter (Figure 3C). Thus, self-organized gut organoids exhibited diverse types of highly differentiated intestinal cells.

Gut organoids show key functional features of mature gut. The gut organoids were analyzed for intestinal functions, namely contractile activity, peptide absorption, and fluid secretion. Gut organoids showed contractile movements, indicating the presence of a functionally mature mesenchymal layer. Gut motility is controlled by the enteric nervous system and pacemaker cells, such as the interstitial cells of Cajal (ICC), which stimulate the slow wave contractions of intestinal smooth muscle cells $(16,17)$. To determine which cell types were responsible for the contractile movements in organoids an immunochemical analysis was performed. Mesoderm-derived smooth muscle cells were identified in the submucosal area by staining for $\alpha$-smooth muscle actin (SMA) expression (Figure 4A); the presence of these cells indicated the contribution of mesenchyme to the formation of organoids. Intestinal subepithelial myofibroblasts support in vitro and in vivo growth of human intestinal epithelium (18). Quantitative RT-PCR was used to identify cells showing expression of protein gene product 9.5 (PGP9.5), a neural marker for the enteric nervous system (Figure $3 \mathrm{~A}$ ), and the ICC markers CD34 and CKIT (Figure 2D). Immunostaining was used to identify cells doubly positive for CKIT and S-100, a glial cell marker; these cells were localized in submucosal regions (Figure 4B). Serotonin is a major neurotransmitter that regulates gastrointestinal functions such as peristalsis and secretion; it is synthesized by enteroendocrine cells of the intestinal mucosa (19). Immunohistochemical analysis revealed that serotonin-positive cells were present in the epithelial layers of gut organoids (Figure 4C). The modulation of contractile activity after treatment with histamine or the muscarinic receptor antagonist atropine (Figure 4D and Supplemental Video 2) confirmed that the gut organoids showed similar motility as mature intestine (20). Histamine treatment increased the rate of gut organoid contractile activity, whereas atropine treatment decreased contraction amplitude and frequency. Motile gut organoids 

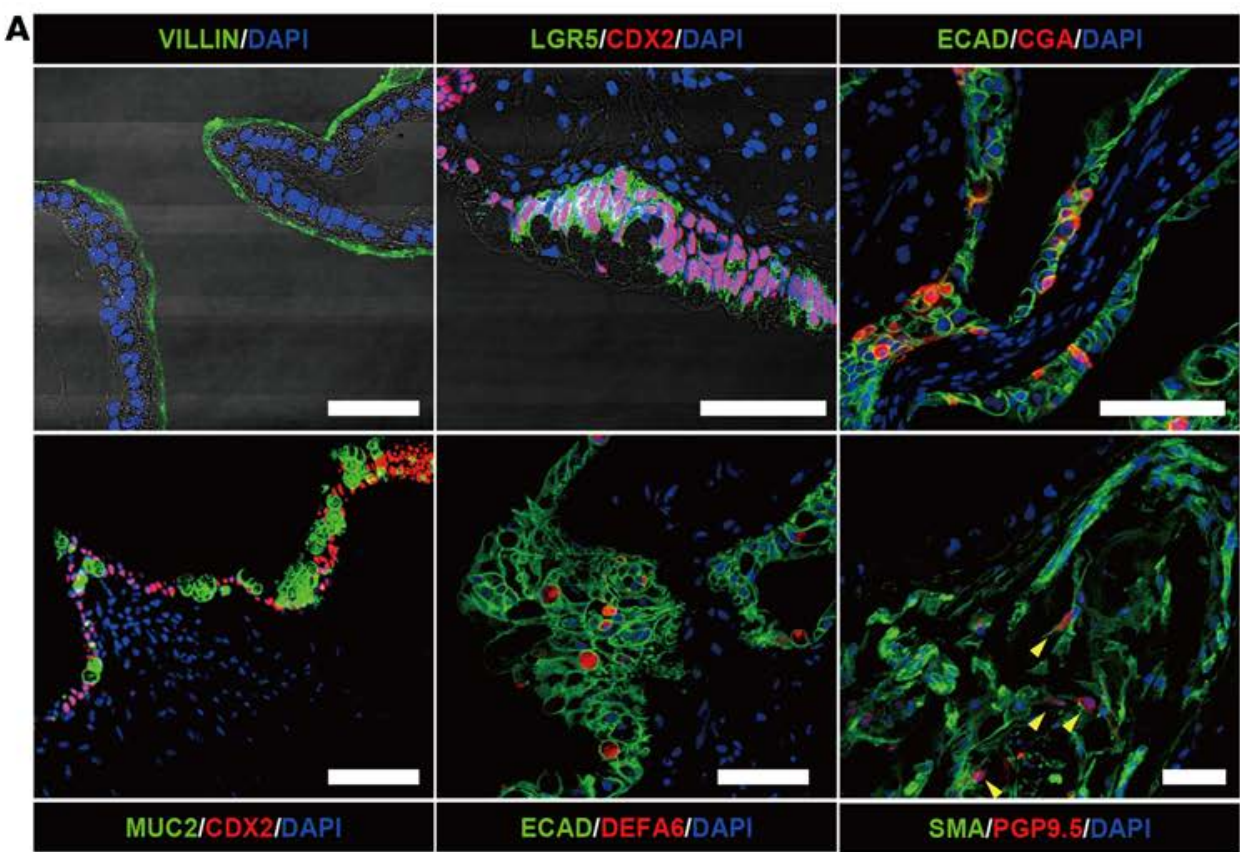

B
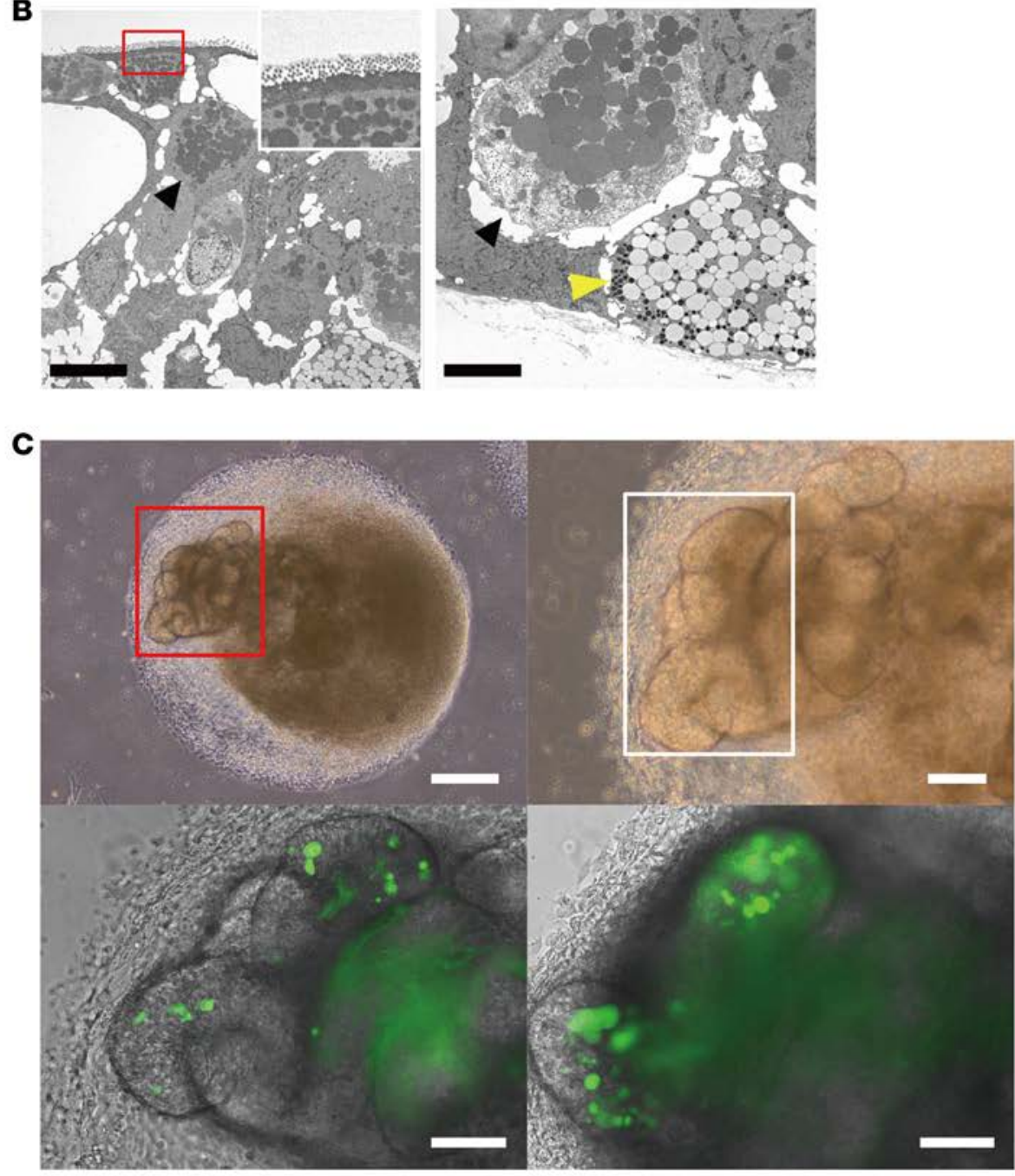

Figure 3. Characterization of gut organoids and detection of LCR5-EGFP-positive cells during gut organogenesis. (A) Day 50-60 organoids from human embryonic stem cells (hESCs) (SEES1 cells) immunostained with markers for intestinal differentiation: villin, leucine-rich repeat containing $G$ protein-coupled receptor 5 (LGR5), CDX2, E-cadherin (ECAD), chromogranin A (CCA), mucin-2 (MUC2), defensin $\alpha-6$, Paneth cell-specific (DEFA6), $\alpha$-smooth muscle actin (SMA), and protein gene product 9.5 (PGP9.5). Cell nuclei were counterstained with DAPI. PGP9.5-positive enteric neuronal cells within the $\alpha$-SMA-positive myenteric area are indicated by yellow arrowheads. Scale bar: $50 \mu \mathrm{m}$ (VILLIN, LGR5, SMA); 100 $\mu \mathrm{m}$ (ECAD and MUC2). (B) An enterocyte with a characteristic brush border (left), and Paneth cells with secretory granules (black arrowhead) and goblet cells containing mucin granules (yellow arrowhead) (right). Scale bar: $10 \mu \mathrm{m}$ (left); $5 \mu \mathrm{m}$ (right). (C) Organoids that developed from SEES1 cells expressed EGFP under the LGR5 promoter (green), indicating that they were LGR5-positive gut organoids. Gut tube-like architecture in a day 34 organoid (red square; high-magnification view, white square) (top row). A small number of EGFP-positive cells were detected in the day 34 organoid (white square) by fluorescence microscopy (bottom left). The number of EGFP-positive cells increased at day 41 (bottom right). Scale bar: $300 \mu \mathrm{m}$ (top left), $100 \mu \mathrm{m}$ (top right and bottom row).

that were contractile also responded to the drugs; however, nonmotile organoids were never observed to respond to the contractile activator histamine ( $n=6$; Figure $4 \mathrm{E}$ and Supplemental Video 3). By contrast, histamine $\mathrm{H} 1$ receptors were expressed in epithelial and mesenchymal lesions in motile organoids and human intestines (Figure 4F). Nonmotile organoids were also characterized as gut-type tissue by quantitative RT-PCR and immunostaining analyses for intestine tissue-specific genes (Supplemental Figure 2A). The markers of mature 
A

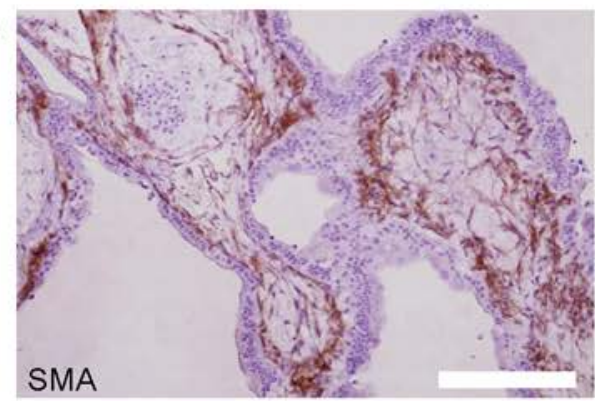

B

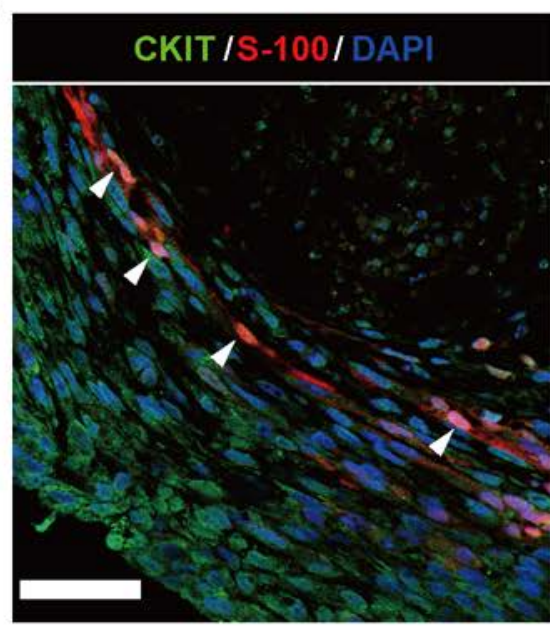

C

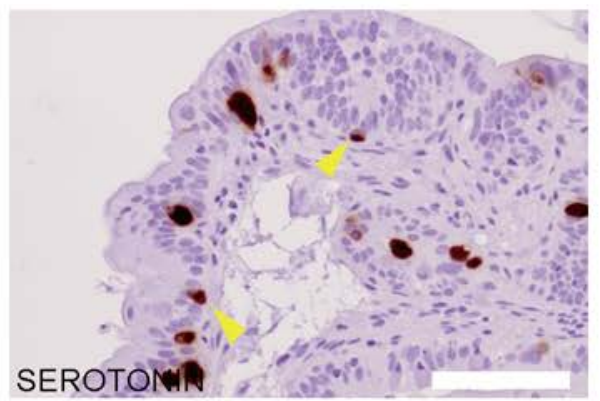

D

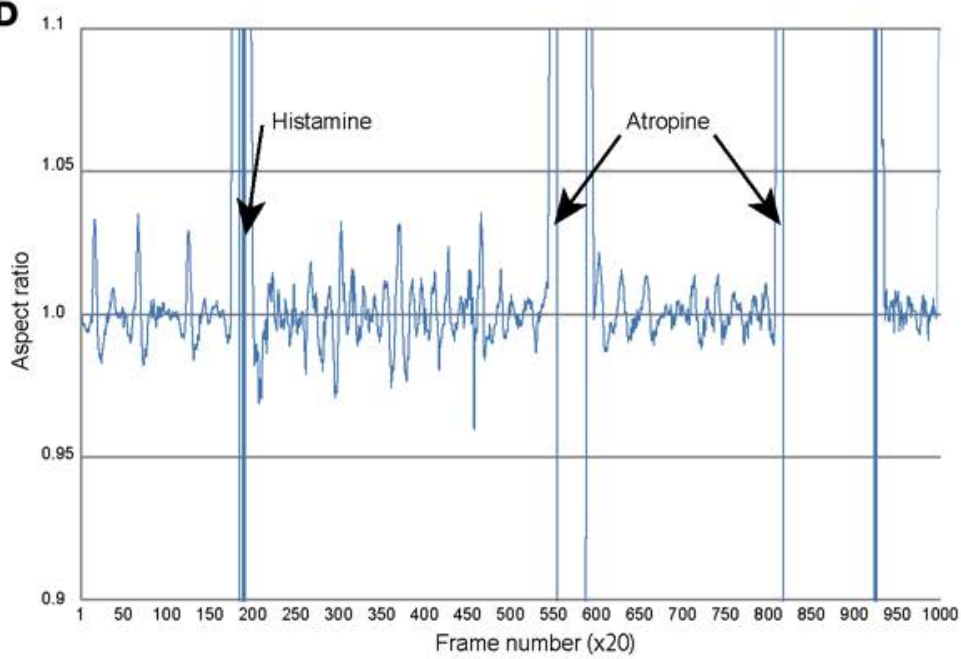

E

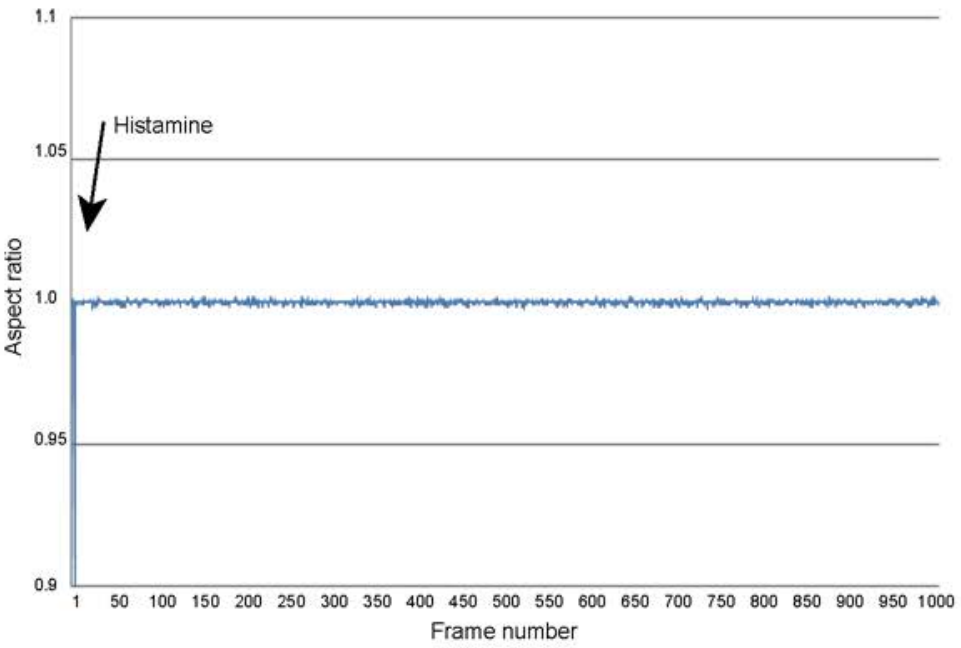

$\mathbf{F}$

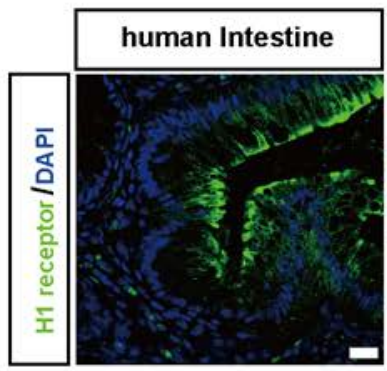

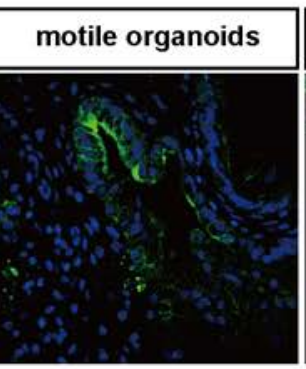

non-motile organoids

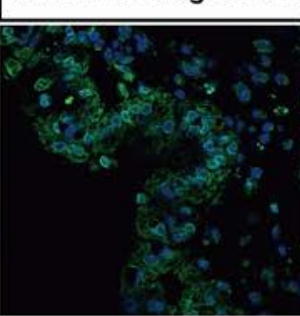

Figure 4. Contractility of gut organoids. (A) Immunohistochemical staining for $\alpha$-smooth muscle actin (SMA) in a day 60 gut organoid. Scale bar: $200 \mu \mathrm{m}$. (B) Immunostaining for the intestinal cells of Cajal. CKIT and S-100 double-positive cells within the myenteric and submucosal plexuses (white arrowheads). Scale bar: $50 \mu \mathrm{m}$. (C) The distribution of the neurotransmitter serotonin, an enteroendocrine cell marker, in a representative hESC-derived day 60 gut organoid. Serotonin-positive cells were observed in the lining epithelium and displayed a triangular shape (yellow arrowheads). Scale bar: $100 \mu \mathrm{m}$. (D) Contractile activity of the motile gut organoid in response to pharmacological agents (Supplemental Video 2). The aspect ratios are based on the ratio of the longest diameter to the shortest diameter of the organoid, which were calculated for each frame. The original video was recorded at 30 frames per second. The playback speed of the video was 20 times actual speed. Waves of constant contractions were observed before treatment (frames 1-160), histamine treatment increased the frequency of gut organoid contractile activity in frequency, and atropine treatment decreased contraction amplitude and frequency. (E) Nonmotile organoids $(n=6)$ did not show contractions in response to histamine (Supplemental Video 3). The original video was recorded at 30 frames per second. (F) Human intestine tissue and motile and nonmotile organoids were immunostained for histamine $\mathrm{H} 1$ receptor. Cell nuclei were counterstained with DAPI. Nonmotile organoids showed positive staining in the epithelial and mesenchymal areas. Scale bar: $20 \mu \mathrm{m}$. 
endoderm derivatives, ALBUMIN and INSULIN, were not expressed in gut organoids (Supplemental Figure 2B). Nonmotile organoids showed similar levels of expression of the tested genes as adult intestine; however, immunostaining analysis showed that the organoids displayed immaturity/disorganization of the mesenchymal layers. The smooth muscle cell marker SMA showed a low level of staining compared with motile organoids (Supplemental Figure 2C). Neurofilaments were distributed throughout the mesenchymal area of motile organoids but were present much more infrequently in nonmotile organoids (Supplemental Figure 2D). Immaturity of muscle cell layers and neurons in the mesenchymal area might be the cause of nonmotility in these organoids.

To examine peptide absorption in gut organoids, the relative expression levels of intestinal oligopeptide transporter (PEPT1) and two major ATP-binding cassette $(A B C)$ transporters, $A B C B 1$ and $A B C G 2$, were screened. Levels of expression in organoids were similar to those in the mature intestine (Figure $5 \mathrm{~A}$ ). Active peptide transport was evaluated in an ex vivo assay used to characterize transport across the mammalian intestine (21). Organoids cultured with the fluorophore-conjugated dipeptide $\beta$-Ala-Lys- $N$ (epsilon)-7-amino-4-methylcoumarin-3-acetic acid showed peptide absorption, which decreased after treatment with the angiotensin-converting enzyme inhibitor captopril (Figure 5B). There was no indication of dose dependency after treatment with different concentrations of captopril (Figure $5 \mathrm{C})$. The inhibitor $(10 \mu \mathrm{M})$ blocked peptide absorption in all organoids we examined. These experiments were performed on three biological replicates for each concentration of captopril $(10 \mu \mathrm{M}, 100 \mu \mathrm{M}$, and $1 \mathrm{mM})$ and were similarly performed for the control without captopril.

In order to examine secretory activity, we screened organoids for expression of the cystic fibrosis conductance regulator (CFTR) and also performed a forskolin-induced swelling (FIS) assay. CFTR is critical to fluid secretion in intestinal epithelial cells. We confirmed the presence of CFTR in epithelial layers of the gut organoids, as well as in control human intestine (Figure 6A). To demonstrate CFTR function, we used the well-established FIS assay (22) and found that gut organoids were enlarged by forskolin induction (Supplemental Video 4); CFTR blockers completely inhibited this swelling (Figure 6B). Our analyses therefore demonstrate that the gut organoids displayed absorptive and secretory activities similar to those of mature intestine.

Taken together, these data indicate that stem cell-derived gut organoids produced in XF conditions have a well-organized structure containing all major intestinal cell lineages and show intestinal functions similar to those of mature intestine. A previous study showed that maturation and differentiation occurred in gut organoids transplanted under mouse kidney capsules (23). To confirm the maturity of the gut organoids derived in vitro from stem cells, single day 35 organoids derived from SEES1 cells and constitutively expressing EGFP under the CMV promoter were transplanted under the renal capsules of adult nude mice (Supplemental Figure $3 \mathrm{~A})$. All transplanted organoids $(n=4)$ successfully engrafted and displayed highly structured intestinal tract morphology, with a lumen and laminated subepithelial layers (Supplemental Figure 3, B and C). We also observed expression of the intestinal epithelial and mesenchymal gene markers $C D X 2$ (enterocytes), $M U C 2$ (goblet cells), CGA (enteroendocrine cells), DEFA6 (Paneth cells), and ECAD and $\mathrm{Na}^{+} / \mathrm{K}^{+}$-ATPase (epithelium) as well as the enteric neuronal marker PGP9.5 in the layered SMA-positive mesenchyme (Supplemental Figure 3D). Thus, all intestinal cell types were present. Transplantation experiments showed that gut organoids did not grow much, indicating that they were highly differentiated in vitro.

\section{Discussion}

The intestine is a complex organ functionally and developmentally. Gastrointestinal tube development involves a series of complex mutual interactions among the mesenchyme, endodermal epithelium, and ectodermal enteric nervous system (3, 4). Many studies on the basic function and clinical gastroenterology of the mature intestine have used cell lines and expanded primary tissues; however, both of these have significant limitations (24). An alternative approach of using cellular organoids is under development and potentially offers a novel tool for in vitro research in this area $(24,25)$. Organoids can be generated from gastrointestinal tissue progenitors or PSCs that differentiate into spatial organ-like tissues exhibiting multiple cell types and functions.

Our results demonstrate that human stem cell-derived gut organoids produced in XF conditions have a well-organized structure and contain all the major intestinal cell lineages. Importantly, these organoids display similar functional characteristics to mature intestine. The gut organoid system has at least three advantages for this type of research. First, gut organoids can be derived and maintained for long-term periods under XF conditions and are relatively stable without the use of xenogeneic-derived materials such 
A

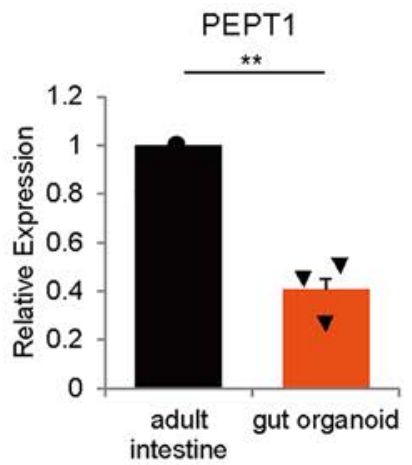

B
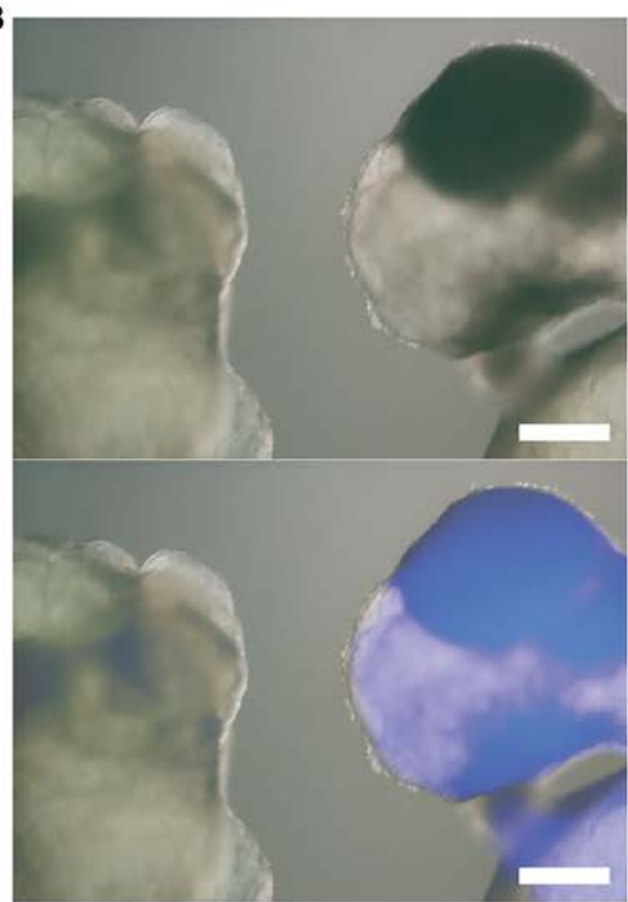

C
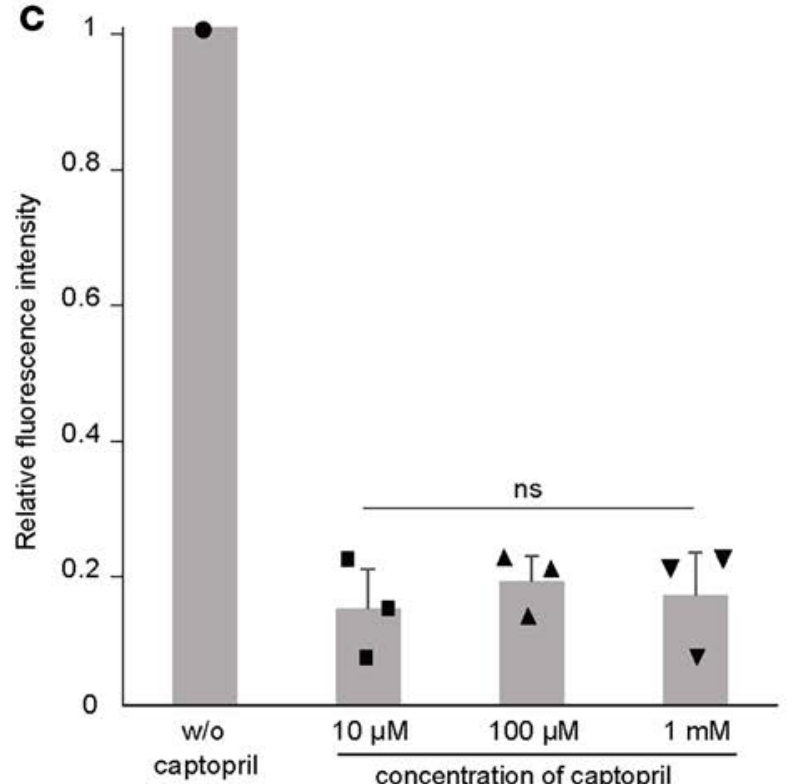

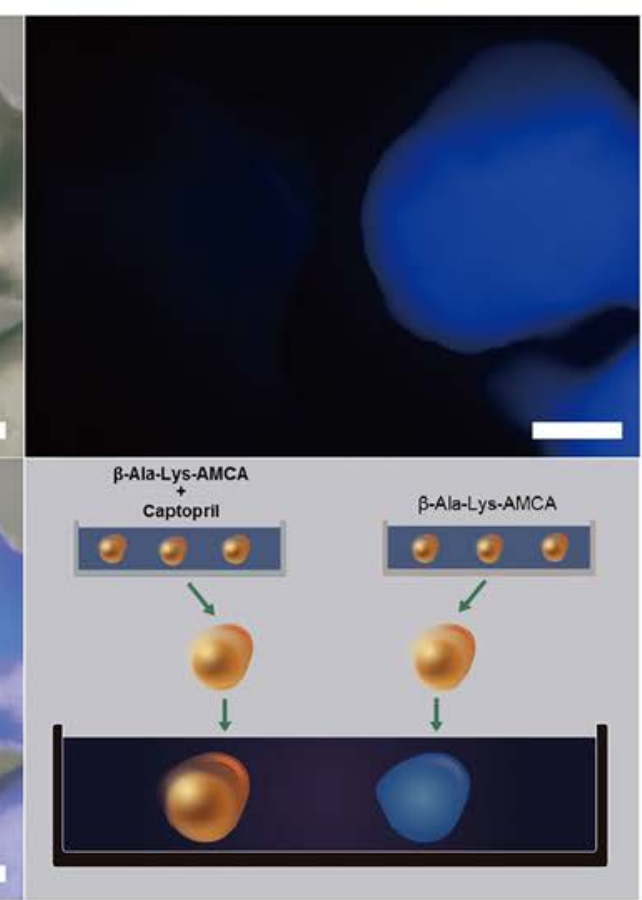

ABCB1
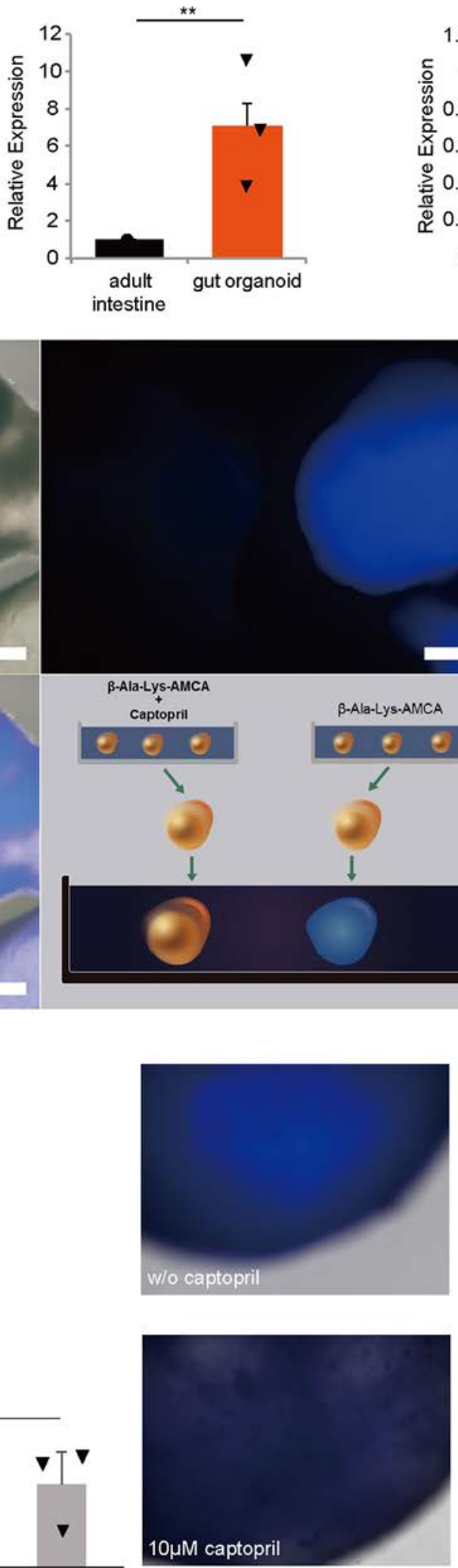

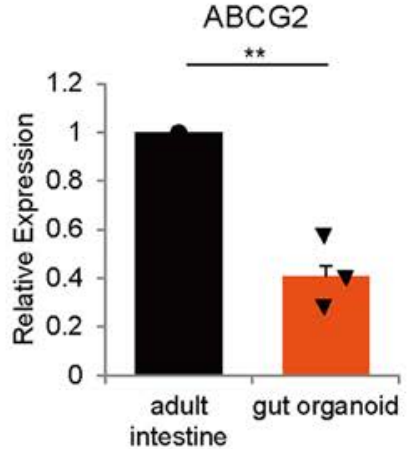

Figure 5. Absorptive functions of gut organoids. (A) The expression of the intestinal oligopeptide transporter (PEPT1) and major ATP-binding cassette (ABC) transporters $A B C B 1$ and $A B C G 2$ in hESC-derived day 50 gut organoids was analyzed by qRT-PCR and compared to that in the healthy adult small intestine. Statistical analysis was performed using a $t$ test or a Mann-Whitney rank-sum test $\left({ }^{* *} P<\right.$ $0.01)$. Data are shown as means (\%) $\pm \mathrm{SEM} ; n=3$. (B) Gut organoids were treated with fluorophore-conjugated dipeptide $\beta$-Ala-Lys-AMCA with or without angiotensin-converting enzyme inhibitor captopril (top row and bottom left). The gut organoids clearly showed dipeptide uptake (top right) inhibited by captopril (top left). Gut organoids are visible in the the bright-field image (top left), the fluorescence image (top right), and the combined bright-field and fluorescence image (bottom left) $(n=3$ for each group). Schematic diagram of the peptide absorption assay (bottom right). Scale bar: $200 \mu \mathrm{m}$. (C) To quantify the uptake of $\beta$-Ala-Lys-AMCA, the AMCA-related signals in gut organoids cultured with or without $10 \mu \mathrm{M}, 100 \mu \mathrm{M}$, and $1 \mathrm{mM}$ of captopril were observed using a fluorescence microscope BZ-X710 (Keyence) equipped a top-stage incubator $\left(5 \% \mathrm{CO}_{2}\right.$ at $37^{\circ} \mathrm{C}$ ) (images on the right), and the fluorescence signal intensity was quantified by using Hybrid Cell Count BZ-H3C (Keyence). The results did not show dose dependency. All sample images were recorded under standard conditions. Each concentration assay was performed with 3 biologically independent replicates. 

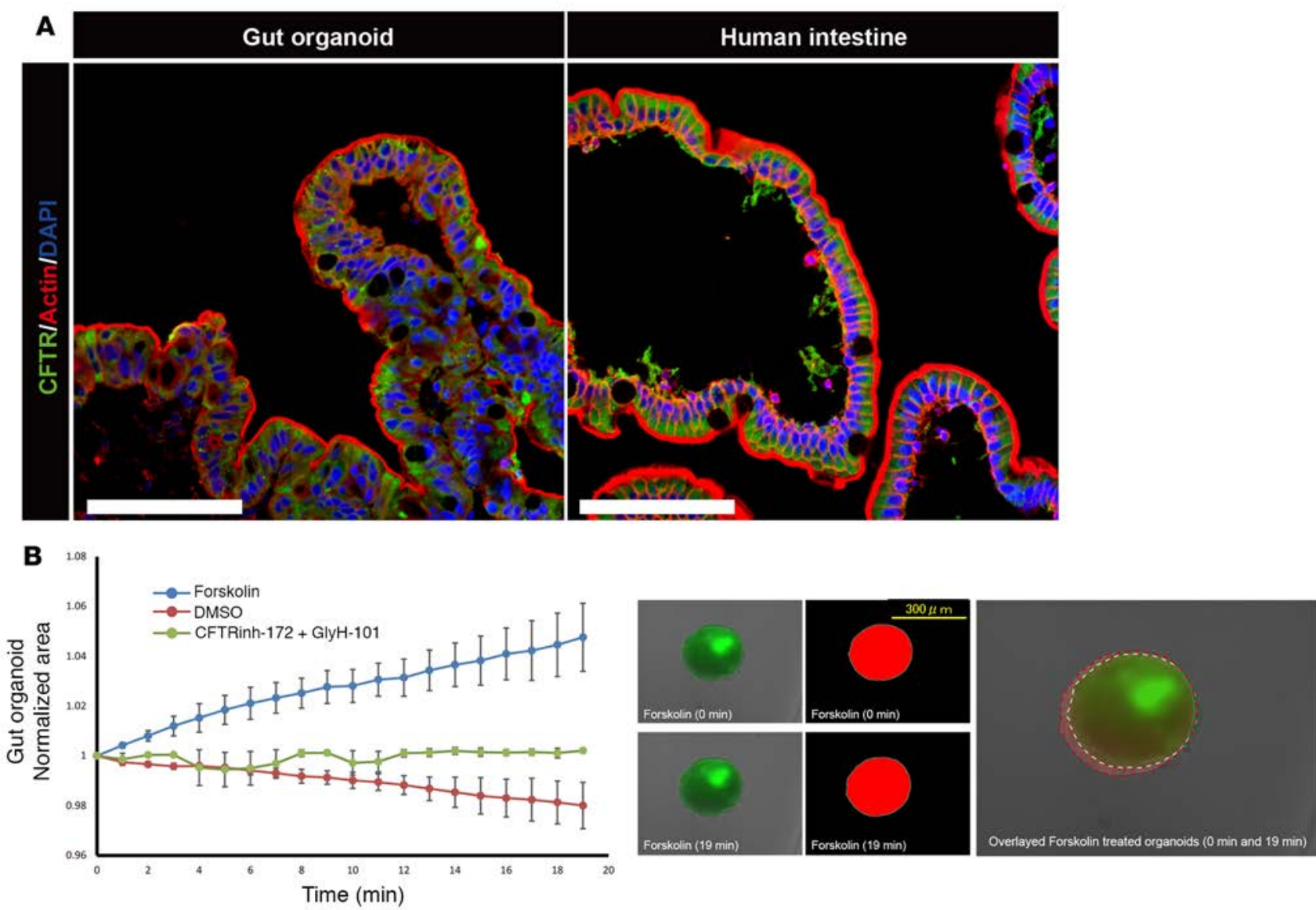

Figure 6. CFTR transport activity of gut organoids. (A) Day 115 gut organoids from hESCs (SEES1 cells) immunostained with markers for the cystic fibrosis conductance regulator (CFTR). CFTR was present in the epithelial layers of the gut organoids as well as in the control human intestine. CFTR (green), actin (red), and DAPI (blue). Scale bar: $100 \mu \mathrm{m}$. (B) Quantification of forskolin-induced gut organoid swelling. Gut organoids (green) derived from SEES1 cells were monitored by time-lapse fluorescence laser confocal microscopy (Keyence). The organoid surface area (red) was quantified using Hybrid Cell Count BZ-H3C (Keyence). The normalized total organoid surface area was calculated and averaged from 3 individual wells per treatment ( $n=3$ for each group). A forskolin-induced organoid (19 minutes) was overlaid with a pretreated organoid ( 0 minutes; $569 \mu \mathrm{m}$ ).

as fetal bovine serum. The XF origin is important for potential future clinical applications in regenerative medicine. Second, gut organoids have a complex tissue architecture and show similar functions as mature intestines. Unlike tissue-derived epithelial organoids, gut organoids contain both intestinal epithelium and mesenchymal layers. Gut organoids show the epithelial functions of peptide absorption and fluid secretion and display peristalsis-like movements in response to histamine and atropine similar to those of mature human intestine. This suggests that gut organoids have mature epithelial and mesenchymal layers and are "miniature guts," like a downsized human intestinal organ. The gut organoid system may be of value for investigations into severe diseases such as inflammatory bowel disease and other congenital intestinal diseases. Third, to our knowledge, this is the first report showing that functional organoids containing all 3 germ layers can be generated from hPSCs in vitro.

Based on the motility of the organoids, a substantial proportion were deemed to be nonmotile, although they still showed epithelial functions. Our immunostaining analysis indicated immaturity of mesenchymal layers such as smooth muscles and neurons. This observation suggests that further improvements will be needed to generate gut organoids with more mature mesenchymal layers.

In conclusion, we have developed a simple, XF system to generate mature differentiated gut organoids from hPSCs without the need for multiple cell passages. Our method could be used to investigate mechanisms underlying intestinal diseases and facilitate the use of gut organogenesis in vitro for clinical and drug development studies for treatment of severe bowel syndrome and other gastrointestinal disorders. 


\section{Methods}

Cell lines. The hESC lines SEES1, SEES2, and SEES3 were generated in our laboratory (13) and were maintained on irradiated mouse embryonic fibroblast (MEF) feeder layers in Knockout DMEM containing 20\% KnockOut Serum Replacement (Life Technologies), $0.1 \mathrm{mM}$ nonessential amino acids (NEAA), $2 \mathrm{mM}$ GlutaMAX-I, $0.055 \mathrm{mM} \beta$-mercaptoethanol, $50 \mathrm{U} / \mathrm{ml}$ penicillin $/ 50 \mu \mathrm{g} / \mathrm{ml}$ streptomycin (Pen-Strep), and $8 \mathrm{ng} / \mathrm{ml}$ recombinant human bFGF (all from Life Technologies, Carlsbad, CA, USA). The medium was changed every two days, and cells were subcultured approximately once a week using enzymatic (dispase; Wako Pure Chemical Industries) or mechanical (EZPassage; Life Technologies) methods. hiPSCs were generated from the fetal lung fibroblast MRC5 cell line in our laboratory (26-28) and maintained on irradiated MEF feeder layers in iPSellon medium (Cardio Incorporated) supplemented with $10 \mathrm{ng} / \mathrm{ml} \mathrm{bFGF}$.

Generation of human gut organoids. In a previous study, we established defined XF conditions for generating and expanding hESCs (13). hESCs were stably maintained in XF hESC culture medium containing 85\% Knockout DMEM, 15\% Knockout Serum Replacement XF CTS (XF-KSR; Life Technologies), 2 mM GlutaMAX-I, 0.1 mM NEAA, Pen-Strep, $50 \mu \mathrm{g} / \mathrm{ml}$ L-ascorbic acid 2-phosphate (Sigma-Aldrich), $10 \mathrm{ng} /$ $\mathrm{ml}$ heregulin-1 $\beta$ (recombinant human NRG- $\beta$ 1/HRG- $\beta 1$ EGF domain; R\&D Systems), $200 \mathrm{ng} / \mathrm{ml}$ recombinant human IGF-1 (LONG R ${ }^{3}$-IGF-1; Sigma-Aldrich), and 20 ng/ml human bFGF (Life Technologies).

To generate gut organoids, undifferentiated hESCs were dissociated using dispase and plated on a cell-patterning glass substrate CytoGraph (Dai Nippon Printing Co. Ltd.), coated with $0.1 \%$ human recombinant type I collagen peptide (RCP) (Fujifilm) in 90-mm culture dishes. Other matrices such as vitronectin (Life Technologies) can also be used for this protocol. Matrix-coated dishes were prewarmed at $37^{\circ} \mathrm{C}$ for 1 hour, the coating solution was removed, and the plates were washed 3 times with PBS. The cells $\left(4 \times 10^{6}\right.$ in $3 \mathrm{ml}$ of culture medium) were plated onto the patterned substrate areas for 10 minutes; the medium was aspirated, and $10 \mathrm{ml}$ of fresh medium was added gently. For the experiments involving the Rho-associated protein kinase inhibitor Y-27632 (Wako Pure Chemical Industries), hESCs or hiPSCs were cultured in XF hESC medium without growth factors (XF-KSR(-)) for 1 day and then in XF-KSR medium, which was replaced after 3 days with the XF hESC medium used as the differentiation medium in the gut organoid protocol. The differentiation medium was gently changed every 3 to 4 days. After 40 days, gut-like peristaltic organoids were collected and cultured together in 60-mm Ultralow Adhesion plates (NOF Corporation) in the differentiation medium.

Video recording of human gut organoids. Floating organoids were picked, placed into culture plates with the differentiation medium, and analyzed by video recording using an inverted microscope (IX71, Olympus) equipped with a camera (Cohu 3600) of ZILOS-tk system (Hamilton Thorne). To count contracting gut organoids, all hiPSC-derived floating organoids from one plate were collected in a new dish and recorded for 10 minutes; peristalsis-like contracting organoids were counted as positive. Because hundreds of organoids were produced in each experiment, it took several hours to observe slowly contracting gut organoids in vitro at room temperature in a normal atmosphere. Therefore, to prevent organoid degradation during recording, we used 10-minute recoding intervals and subsequently placed the cells back in the incubator. Three independent experiments were performed; in total, 791 floating organoids were analyzed.

Quantitative RT-PCR analysis. RNA was isolated from organoids using the RNeasy Mini Kit (Qiagen), and contaminating DNA was removed using DNase (Life Technologies). First-strand cDNA was synthesized using SuperScript III reverse transcriptase and an oligo-dT primer (Life Technologies) according to the manufacturer's instructions. Quantitative RT-PCR was performed in triplicate using the SYBR Green PCR Master mix and a QuantStudio 12K Flex Real-Time PCR System (Life Technologies); primer sequences are listed in Supplemental Table 1. After amplification, dissociation curves were obtained to ensure that every PCR product was amplified. QuantStudio 12K Flex software v1.0 was used to quantify the relative levels of mRNA of the target genes after normalization against the housekeeping gene GAPDH. Healthy human adult small intestinal tissue (R1234226-50, BioChain Institute) and human adult pancreas and liver cDNAs (HA188 and HA-149, respectively, Alpha Diagnostic International) were used as a positive controls.

Immunocytochemistry. Organoids were fixed with $4 \%$ paraformaldehyde in PBS (Wako Pure Chemical Industries) for 5 minutes at $4^{\circ} \mathrm{C}$, permeabilized with $0.2 \%$ Triton X-100 for 2 minutes at room temperature, and blocked with PBS containing 5\% normal serum as appropriate for each antibody. The cells were then incubated overnight at $4^{\circ} \mathrm{C}$ with primary antibodies against villin (sc-7672, 1:50, Santa Cruz Biotechnology); E-cadherin (610181, 1:50, BD Pharmingen); CGA (ab16007, 1:100), CDX2 (ab76541, 1:100), and PGP9.5 (ab8189, 1:10) (from Abcam); DEFA6 (HPA019462, 1:500) and SMA (A2547, 1:400) (from Sigma-Aldrich); 
MUC2 (sc-7314, 1:50, Santa Cruz Biotechnology); LGR5 (LMC-1235, 1:100, Medical \& Biological Laboratories); CKIT (NB100-77477AF488, 1:10, Immuno-Biological Laboratories); $\mathrm{Na}^{+} / \mathrm{K}^{+}$-ATPase (NB300146, 1:100, Novus Biologicals); S-100 (422091, 1:100, Nichirei Biosciences); neurofilament (M076229, 1:50, Dako); $\beta$-actin (A5316, 1:1,000, Sigma-Aldrich); histamine H1 receptor (aa471-484, 1:200, LSBio); and CFTR (ab131553, 1:100, Abcam). Alexa 488- or Alexa 546-conjugated anti-mouse, anti-rabbit, or anti-goat secondary antibodies (BD Biosciences) were used; nuclei were counterstained with DAPI. Cell fluorescence was analyzed using an LSM 510 Meta Laser Scanning Confocal Microscope (Carl Zeiss Microscopy).

Histochemical analysis. Organoids were fixed in 4\% paraformaldehyde, embedded in paraffin, and serially sectioned into 4- $\mu \mathrm{m}$ sections. Alternate sections were mounted on slides, stained with H\&E, and analyzed. For Alcian Blue staining, sections were incubated in 1\% Alcian Blue in 3\% acetic acid, pH 2.5, for 20 minutes and in $0.1 \%$ nuclear Fast Red for 2 minutes, dehydrated in ethanol, and cleared using xylene.

Immunohistochemical analysis of developed organoids was carried out using sections stained with antibodies against SMA and serotonin (ab16007, 1:20, Abcam). The secondary antibody was polyclonal rabbit anti-mouse immunoglobulin $\operatorname{HRP}$ (1:100; Dako).

Electron microscopy. Gut organoid samples for electron microscopy were prepared according to a standard protocol (Tokai Electron Microscopy). Briefly, organoids were fixed with 2\% paraformaldehyde and $2 \%$ glutaraldehyde in PBS, dehydrated, and embedded in the epoxy resin. After polymerization, the samples were sectioned at $70 \mathrm{~nm}$, mounted on copper grids, and observed under a JEM-1200EX TEM (Jeol Ltd.) equipped with a Veleta CCD camera (Olympus).

Cell transfection. To monitor differentiation status during gut organogenesis, we generated an intestinal lineage-specific reporter construct (pPB-hLgr5p-EGFP-neo) containing the 5-kb LGR5 promoter and the phosphoglycerine kinase $(P G K)$ promoter to drive expression of EGFP and the neomycin resistance gene (neo), respectively. SEES1 cells were cultured with Y-27632 for 24 hours prior to electroporation, washed with PBS, harvested using Accutase solution (Life Technologies), and resuspended in iPSellon medium. Cells were dissociated into a single-cell suspension by vigorous pipetting; $1 \sim 2 \times 10^{6}$ cells were pelleted and mixed with the pPB-hLgr5p-EGFP-neo reporter vector and the hyperactive PiggyBac transposase expression vector (pCMV-hyPBase) (a gift from A. Bradley, Wellcome Trust Sanger Institute, Hinxton, Cambridge, United Kingdom) in Opti-MEM (Life Technologies). The cell suspension was transferred to a cuvette and electroporated using the NEPA21 Super Electroporator (Nepa Gene); transfected cells were selected with G418 (Sigma-Aldrich).

To visualize the in vivo performance of transplanted gut organoids, SEES1 cells were transfected with the constitutive EGFP expression vector (pmGENIE-EGFP) (a gift from S. Moisyadi, University of Hawaii, Honolulu, Hawaii, USA). After screening, a stable GFP-positive SEES1 line was established.

In vitro $\beta$-Ala-Lys-AMCA uptake by human gut organoids. Gut organoids grown in differentiation medium were washed with PBS and cultured in DMEM containing $25 \mu \mathrm{M}$ fluorophore-conjugated dipeptide $\beta$-Ala-Lys-AMCA (Biotrend Chemicals) for 4 hours at $37^{\circ} \mathrm{C}$. For inhibition experiments, gut organoids were cultured with $\beta$-Ala-Lys-AMCA with or without $1 \mathrm{mM}$ of angiotensin-converting enzyme inhibitor captopril (Sigma-Aldrich) for 1 hour, rinsed with PBS, placed into the same dish, and observed under a fluorescence microscope (Olympus). In addition, to quantify the uptake of $\beta$-Ala-Lys-AMCA, gut organoids were cultured with or without $10 \mu \mathrm{M}, 100 \mu \mathrm{M}$, or $1 \mathrm{mM}$ of captopril, and AMCA-related signals were observed using a fluorescence microscope (BZ-X710; Keyence) equipped a top-stage incubator $\left(5 \% \mathrm{CO}_{2}\right.$ at $\left.37^{\circ} \mathrm{C}\right)$, and the fluorescence signal intensity was quantified using the Hybrid Cell Count/BZ-H3C (Keyence). All sample images were recorded under standard conditions. Assays for each concentration were performed using 3 independent biological samples.

In vitro contractility of human gut organoids. A single hESC-derived gut organoid showing peristalsis-like movement (day 80-90) was treated with the peristaltic stimulator histamine $(0.2 \mu \mathrm{M})$ and the cholinergic antagonist atropine sulfate $(0.2 \mu \mathrm{M})$; contractility responses were recorded using an inverted microscope. Image analysis software CL-Quant version 3.10 (Nikon Corporation) was used to visualize movements in the gut organoid. First, in each frame of the time-lapse images, the software was used to identify a specific region of the organoid. Second, the software fitted an ellipse to this region and calculated the ratio of the longest to shortest diameter to obtain an aspect ratio. Changes in the aspect ratios with time were plotted on the chart. Videos were recorded at 30 frames per second.

CFTR transport activity of gut organoids. Each organoid was placed into a well of an ART culture dish 12 (NIPRO) containing $100 \mu \mathrm{l}$ culture medium. Organoids derived from EGFP-SEES1 cells, which constitu- 
tively express EGFP under the CMV promoter, were used to visualize volume changes. Five $\mu \mathrm{M}$ forskolin was added, and organoid morphology was monitored by time-lapse fluorescence laser confocal microscopy (Keyence). To inhibit CFTR, organoids were preincubated with $50 \mu \mathrm{M}$ of the CFTR inhibitor CFTRinh172 and $50 \mu \mathrm{M}$ GlyH-101 (TOCRIS) for 3 hours. Images were collected every minute for 20 minutes in a topstage incubator $\left(5 \% \mathrm{CO}_{2}\right.$ at $\left.37^{\circ} \mathrm{C}\right)$. Each experimental treatment was assessed in triplicate wells. DMSO concentration was identical under all conditions and did not exceed $0.2 \%(\mathrm{w} / \mathrm{v})$. The organoid surface area was quantified using Hybrid Cell Count/BZ-H3C (Keyence). The normalized total organoid surface area was calculated and averaged from 3 individual wells per treatment.

Transplantation of human gut organoids. The in vivo development of gut organoids was assessed by transplantation into severe combined immunodeficient nude mice (BALB/cAJcl-nu/nu) purchased from CLEA Japan. A single day 35 gut organoid was transplanted below the kidney capsule of each mouse $(n=4)$ using a mouth-controlled pipette. The transplanted mice were humanely euthanized by cervical dislocation after 6 weeks, and the kidneys were examined under an MVX10 fluorescence stereomicroscope (Olympus). The engrafted site was further analyzed by H\&E staining and immunocytochemistry.

Statistics. Quantitative data are reported as mean \pm SEM from at least 3 independent experiments. Statistical analysis was performed using either an unpaired, 2-tailed $t$ test or a Mann-Whitney rank-sum test. $P$ $<0.05$ was considered to indicate a statistically significant difference.

Study approval. hESC experiments were performed following the hESC guidelines of the Japanese government and were approved by the IRB on hESC research at the National Centre for Child Health and Development (notification no. 232). Surgical specimens for analysis were obtained from patients after informed consent. Parental written informed consent was obtained from all families. The use of intestinal tissues was approved by the IRB of the National Centre for Child Health and Development (IRB permission no. 927), in adherence to the Declaration of Helsinki.

The animal use protocol was approved by the Institutional Animal Care and Use Committee of the National Research Institute for Child Health and Development (permit no. A2003-002). All animal experiments were based on the $3 \mathrm{R}$ principle (refine, reduce, and replace), and all efforts were made to minimize animal suffering and to reduce the number of animals used.

\section{Author contributions}

HA conceived, designed, and coordinated the study and wrote the paper. HU designed the study, performed the analyses, analyzed all the data, and contributed to writing the paper. MM and HU produced the organoids. MM and HA performed the animal surgery. TM prepared the plasmid samples for cell transfections. TK performed some of the experiments and helped prepare the figures. TO developed and provided patterning substrates. KS and MK coordinated human intestinal tissue sample collections under IRB approval. SS and MK participated in the protocol development and data interpretation. NO provided expertise for analyzing gut organoids. AU conceived and coordinated the study and provided expertise on histological analysis.

\section{Acknowledgments}

We are grateful to Dieter Egli and Ben Stanger for critical reading of the manuscript and helpful discussions. We also thank Kenji Osafune of Kyoto University for sharing of primer information for ALBUMIN and INSULIN and Yoshitaro Nakano of NIKON Corporation for quantification analysis of organoid contractions. We thank Minoru Ichinose for preparing histological samples. We thank the members of our laboratories for helpful discussions. This work was supported by grants from the Ministry of Health, Labour and Welfare Sciences to HA and AU; grants from the National Center for Child Health and Development (26-1 and 24-6) to HA; and a grant from JST-CREST to HA. The funders had no role in study design, data collection and analysis, decision to publish, or preparation of the manuscript.

Address correspondence to: Hidenori Akutsu, Department of Reproductive Biology, Centre for Regenerative Medicine, National Research Institute for Child Health and Development, 2-10-1 Okura, Setagaya, Tokyo 157-8535, Japan. Phone: 81.3.5494.7047; E-mail: akutsu-h@ncchd.go.jp.

1. Gracz AD, Magness ST. Defining hierarchies of stemness in the intestine: evidence from biomarkers and regulatory pathways Am J Physiol Gastrointest Liver Physiol. 2014;307(3):G260-G273.

2. Spence JR, Lauf R, Shroyer NF. Vertebrate intestinal endoderm development. Dev Dyn. 2011;240(3):501-520. 
3. Wiginton DA. Gene regulation: The key to intestinal development. In: Sanderson IR, Walker WA, eds. Development of the Gastrointestinal Tract. Hamilton, Ontario, Canada: BC Decker; 1999: 13-36.

4. Xu H, Collins JF, Ghishan FK. Molecular physiology of gastrointestinal function during development. In: Johnson LR, ed. Physiology of the Gastrointestinal Tract. 5th ed. Oxford, UK: Elsevier; 2012: 415-450.

5. Pagliuca FW, Melton DA. How to make a functional $\beta$-cell. Development. 2013;140(12):2472-2483.

6. Zhu Z, Huangfu D. Human pluripotent stem cells: an emerging model in developmental biology. Development. 2013;140(4):705-717.

7. Pagliuca FW, et al. Generation of functional human pancreatic $\beta$ cells in vitro. Cell. 2014;159(2):428-439.

8. Kriks S, et al. Dopamine neurons derived from human ES cells efficiently engraft in animal models of Parkinson's disease. Nature. 2011;480(7378):547-551.

9. Spence JR, et al. Directed differentiation of human pluripotent stem cells into intestinal tissue in vitro. Nature. 2011;470(7332):105-109.

10. Sato T, et al. Single Lgr5 stem cells build crypt-villus structures in vitro without a mesenchymal niche. Nature. 2009;459(7244):262-265.

11. McCracken KW, et al. Modelling human development and disease in pluripotent stem-cell-derived gastric organoids. Nature. 2014;516(7531):400-404.

12. Sasai Y. Cytosystems dynamics in self-organization of tissue architecture. Nature. 2013;493(7432):318-326.

13. Akutsu H, et al. Xenogeneic-free defined conditions for derivation and expansion of human embryonic stem cells with mesenchymal stem cells. Regen Ther. 2015; 1: 18-29.

14. Okochi N, Okazaki T, Hattori H. Encouraging effect of cadherin-mediated cell-cell junctions on transfer printing of micropatterned vascular endothelial cells. Langmuir. 2009;25(12):6947-6953.

15. McGrath KE, Koniski AD, Maltby KM, McGann JK, Palis J. Embryonic expression and function of the chemokine SDF-1 and its receptor, CXCR4. Dev Biol. 1999;213(2):442-456.

16. Sanders KM, Koh SD, Ward SM. Interstitial cells of cajal as pacemakers in the gastrointestinal tract. Annu Rev Physiol. 2006;68:307-343.

17. Huizinga JD, Lammers WJ. Gut peristalsis is governed by a multitude of cooperating mechanisms. Am J Physiol Gastrointest Liver Physiol. 2009;296(1):G1-G8.

18. Lahar N, et al. Intestinal subepithelial myofibroblasts support in vitro and in vivo growth of human small intestinal epithelium. PLoS One. 2011;6(11):e26898.

19. Mawe GM, Hoffman JM. Serotonin signalling in the gut--functions, dysfunctions and therapeutic targets. Nat Rev Gastroenterol Hepatol. 2013;10(8):473-486.

20. Mittal RK, Padda B, Bhalla V, Bhargava V, Liu J. Synchrony between circular and longitudinal muscle contractions during peristalsis in normal subjects. Am J Physiol Gastrointest Liver Physiol. 2006;290(3):G431-G438.

21. Groneberg DA, Döring F, Eynott PR, Fischer A, Daniel H. Intestinal peptide transport: ex vivo uptake studies and localization of peptide carrier PEPT1. Am J Physiol Gastrointest Liver Physiol. 2001;281(3):G697-G704.

22. Dekkers JF, et al. A functional CFTR assay using primary cystic fibrosis intestinal organoids. Nat Med. 2013;19(7):939-945

23. Watson CL, et al. An in vivo model of human small intestine using pluripotent stem cells. Nat Med. 2014;20(11):1310-1314

24. Dedhia PH, Bertaux-Skeirik N, Zavros Y, Spence JR. Organoid Models of Human Gastrointestinal Development and Disease. Gastroenterology. 2016;150(5):1098-1112.

25. Lancaster MA, Knoblich JA. Organogenesis in a dish: modeling development and disease using organoid technologies. Science. 2014;345(6194):1247125.

26. Makino H, et al. Mesenchymal to embryonic incomplete transition of human cells by chimeric OCT4/3 (POU5F1) with physiological co-activator EWS. Exp Cell Res. 2009;315(16):2727-2740.

27. Nishino K, et al. Defining hypo-methylated regions of stem cell-specific promoters in human iPS cells derived from extra-embryonic amnions and lung fibroblasts. PLOS ONE. 2010;5(9):e13017.

28. Toyoda M, et al. Lectin microarray analysis of pluripotent and multipotent stem cells. Genes Cells. 2011;16(1):1-11. 